\title{
Specific Binding of Secondary Antibodies to Neurons in Rat Brainstem Sections
}

\author{
J. Pflugheber, S. Casella, J.S. Erlichman, and T. Budd \\ Department of Biology, St. Lawrence University, 23 Ramoda Dr., Canton, N.Y. 13617, \\ USA
}

Positive and negative controls are an integral part of valid experimental design in fluorescence microscopy. Specimens prepared for immunofluorescence (IF) imagery require a control to test for binding of the fluorescently labeled secondary antibody $(\mathrm{Ab})$ without addition of the primary $\mathrm{Ab}$. To minimize the non-specific secondary Ab binding, many immunological protocols employ a step to block open immunoglobulin receptor sites. Frequently, this blocking step is achieved by incubation of the specimen in serum from the secondary $\mathrm{Ab}$ host species. Alternate methods of blocking open binding sites employ the use of bovine serum albumin or commercially available blocking reagents, however, we have found in brainstem sections that these blocking procedures are ineffective.

Brainstem cryosections were prepared from adults rats that were transcardially perfused with Streck (Streck Laboratories) tissue fixative. Sections were permeabilized by incubation in $0.3 \%$ Triton-X in PBS, followed by blocking with $10 \%$ normal goat serum (NGS). Sections were then incubated in $10 \mathrm{ug} / \mathrm{ml}$ goat anti-mouse (GaM) Alexa 488 for 3 hours, followed by 30' incubation in 50uM Draq-5 (Biostatus LTD), a nuclear stain. Slides were mounted with Fluormount G and visualized on a Leica SP2 confocal laser scanning microscope. The pattern of staining observed suggested the secondary Abs were binding specifically to neurons. This localization was confirmed by incubating sections with mouse anti-NeuN conjugated to Alexa 488, followed by incubation of the section in goat anti-rabbit (GaR) Alexa 546 alone (figure 1).

The staining pattern was unchanged with type of fluor used, blocking reagent, secondary $\mathrm{Ab}$ host species, and fixative. The host of the primary Ab did show differential staining. GaR secondary gave the same strong pattern of staining, while goat anti-chicken secondary gave no background staining (figure 2 ).

On-going experiments are planned to test whether antigen retrieval protocols using various $\mathrm{pH}$ buffers can relieve the specific binding of secondary fluorescenated antibodies to neurons in these sections. It is important to be able to resolve this issue in order to accurately interpret data collected from IF imagery of brainstem tissues.

The authors thank Amy Higgins for providing us with brain sections.

We gratefully acknowledge support for this work from NSF MRI award \# 0116408. 


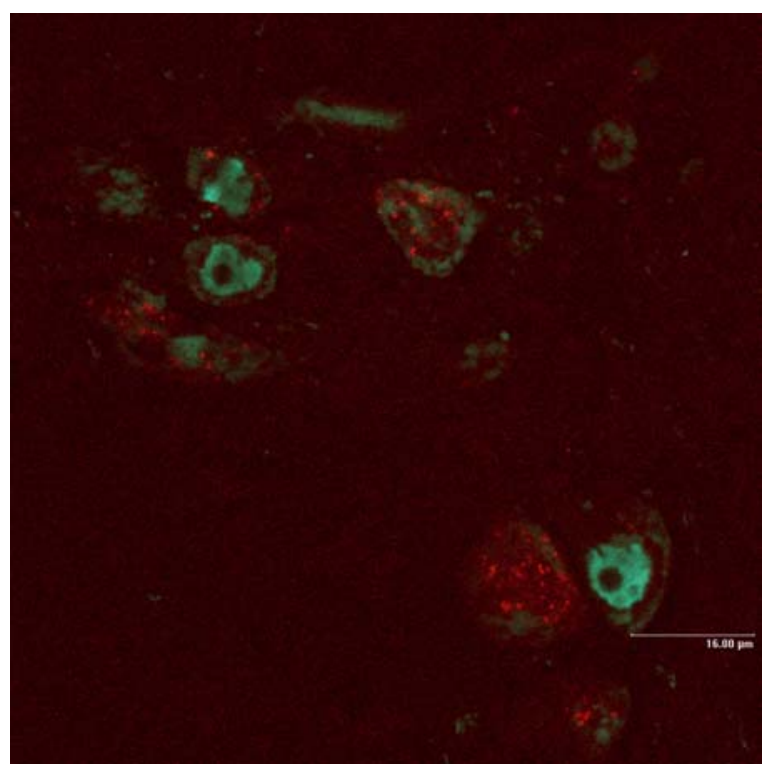

Figure 1.Localization of secondary Ab binding to neurons. Neuronal nuclei are visualized after incubation with Mouse anti NeuN conjugated to Alexa 488 (green). Sections are then incubated with GaR Alexa 546 (red). No Draq-5 was added.

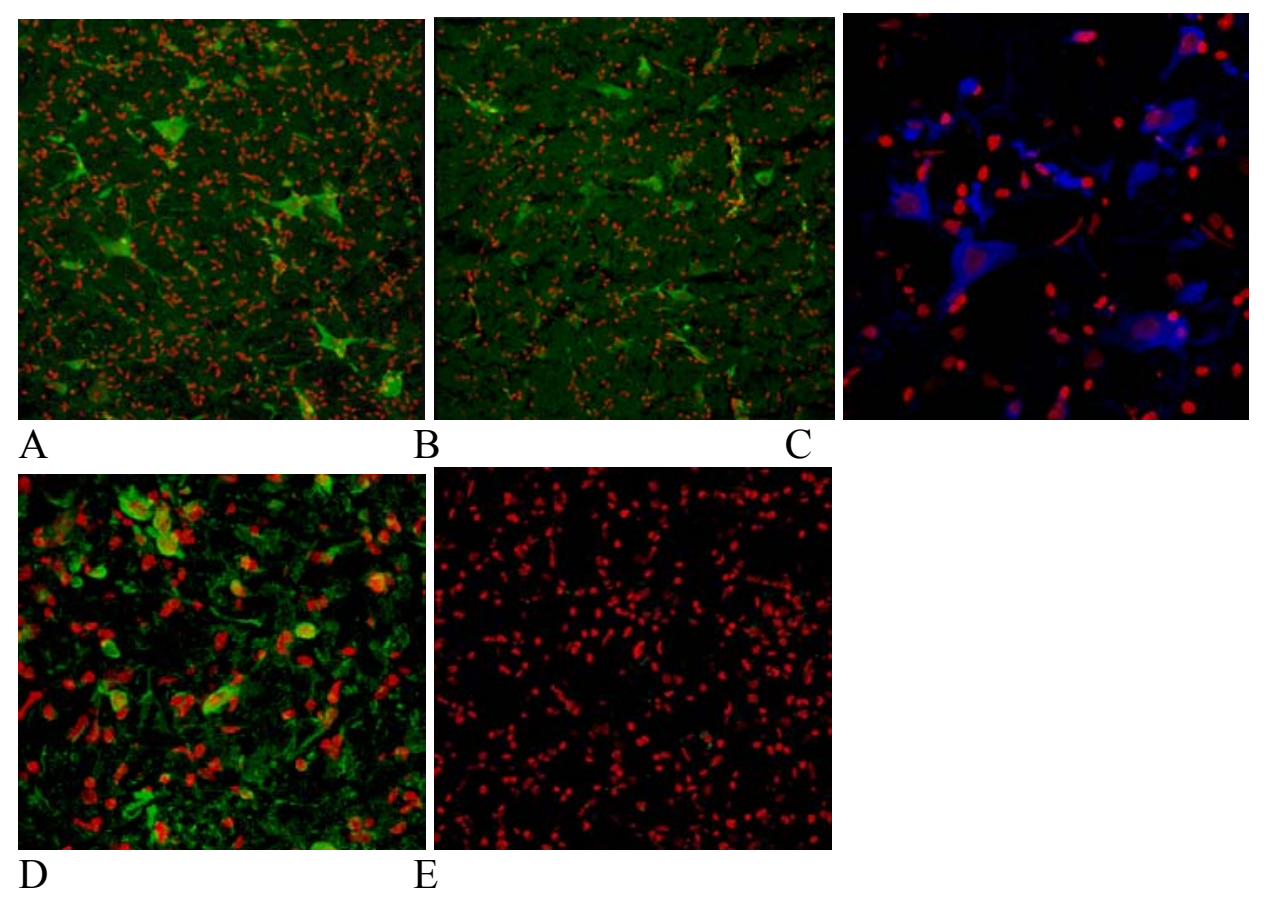

Figure 2. Secondary Ab alone binding. All sections were stained with $10 \mathrm{ug} / \mathrm{ml} \mathrm{Ab}$ diluted in $0.03 \%$ Triton X in PBS, $0.1 \%$ BSA. Nuclei (red) were stained with Draq-5 (50uM). A) GaM Alexa 488 (green) B) GaR Alexa 488 (green) C) GaM Alexa 546 (blue) D) RaM FITC (green) E) goat anti-chicken Alexa 488. 